Tx, is used to measure the velocity of two. An IF signal is calculated by mixing the transmitted and received signals. Each IF signal undergoes an FFT to find a peak corresponding to the range. A second FFT transforms the set of RangeFFT results so that two objects can be distinguished by their phase displacements, which correspond to their different velocities. Therefore, the heart rate can be distinguished from the breathing rate. (a) A frame of transmitted signals are used to (b) detect the range of the chest, which can be (c) transformed into heart rate and breathing rate using FFTs. The IWR1443 mmWave radar sensor obtains heart rate measurements by detecting miniscule movements of the chest caused by the heart's pulse and breathing.

Results A Graphical User Interface was developed using C and MATLAB to display a cardiac waveform and a graph of heart rate values in apparent real-time. Experiments using moving averages were performed to increase the accuracy of the sensor's values.

Conclusion This relatively new mmWave radar technology can be used fairly accurately for the pulse rate monitoring in neonates, children, burns patients or for sleep studies. Being contactless, it prevents skin damage and burns. We recommend the 'Paediatrics 2040' project to encourage the Use and further Research of such monitoring devices to decrease risks.

\section{G92(P) INTER-PROFESSIONAL TRIPARTITE ALLIANCE TO REDUCE MEDICATION ERRORS IN CHILDREN}

${ }^{1} \mathrm{D}$ Paccagnella, ${ }^{2} \mathrm{R}$ Issac, ${ }^{3} \mathrm{~B}$ Patel, ${ }^{1} \mathrm{P}$ Vallabhaneni. 'Paediatrics Department, Swansea Bay University Health Board, Morriston Hospital, Swansea, UK; ${ }^{2}$ Nursing and Midwifery Department, Swansea Bay University Health Board, Morriston Hospital, Swansea, UK; ${ }^{3}$ Pharmacy Department, Swansea Bay University Health Board, Morriston Hospital, Swansea, UK

\subsection{6/archdischild-2020-rcpch.73}

Aims Healthcare professionals are faced with a number of challenges when prescribing and administering medication to children, due to the complexities of age-related drug calculations. Internationally, multiple studies have shown that the regular occurrence of such errors carries a high potential for unintended harm to patients, with research suggesting that good quality care depends upon different professions working together.

In light of this, we reasoned that reducing medication errors could be achieved by multidisciplinary education. For this purpose, a paediatric tripartite alliance between Medicine, Nursing and Pharmacology was created.

Methods An initial prospective audit was undertaken, with the aim of recording the number, nature and severity of reported medication errors over a three - month period. Errors were identified using the Datix database and were classified according to the EQUIP study model.

Following the initial audit, a structured educational programme was introduced to all medical and nursing staff. Subsequently, data was collected over two separate three-month periods.

Results During the pre-intervention period, a total of 159 children were admitted to the paediatric inpatient wards. The total number of recorded medication errors was 142 .

During the first post-intervention period, a total of 470 children were admitted and 57 errors were recorded. Compared with the pre-intervention data, where $89.3 \%$ of admitted patients had been subjected to errors, this value decreased to $12.1 \%$, with an estimated error reduction of $77.2 \%$.

During the second post-intervention period, a total of 338 patients were admitted, with 120 errors recorded (35.5\%).

Conclusions The commitment to tackle the issue of medication errors as part of an inter-professional tripartite alliance, coupled with the implementation of a structured programme, have contributed to a significant reduction in medication errors.

Further improvement could be achieved by understanding and targeting specific environmental and human factors, and by addressing any unmet training needs.

We believe that implementation of similar educational programmes on a wider scale would be achievable and costeffective.

\section{G93(P) FOLLOW UP SKELETAL SURVEY IN CHILD PROTECTION MEDICALS: A QUALITY IMPROVEMENT PROJECT}

L Shipley, A Omokanye, V Walker. Community Paediatrics, Sherwood Forest NHS Trust, Sutton-in-Ashfield, UK

\subsection{6/archdischild-2020-rcpch.74}

Introduction Skeletal fractures are estimated to occur in half of children who have suffered physical abuse. ${ }^{1}$ Fractures secondary to non-accidental injury are often occult and risk under detection if single skeletal survey is undertaken; hence follow up is recommended at 11 to 14 days. $^{2}$ Following a serious case review, quality improvement (QI) service changes were implemented aimed at standardising and simplifying logistics, engaging parents/social services at initial survey, reducing reliance on on-call teams and improving communication to reduce human and system errors.

We evaluated the number of patients who met national criteria for follow up skeletal survey and timing of follow up, if undertaken, both pre and post QI changes.

Method Retrospective audit of children undergoing follow up skeletal survey comparing the rate of follow up within 11-14 days and up to 28 days between June 2017 and June 2019. Data were collected from electronic hospital records and case notes and analysed using Microsoft Excel and Stata with significance as $\mathrm{p}<0.05$.

Results 53 children were included in the audit (20 prechange and 33 post change). QI changes to provision of service resulted in significantly less children missing follow up skeletal survey $(45 \% \quad(n=9) \quad$ vs $3 \% \quad(n=1), p=0.002)$. $68.7 \%(n=22)$ of follow up surveys occurred within the gold standard of 11 to 14 days following QI change compared to $45 \% \quad(n=5)$ prior. Of children who underwent skeletal survey $45 \%$ presented with bruising and 17\% with fracture.

Conclusion Follow-up skeletal survey is an essential part of the child protection medical pathway, occurring ideally within 11 to 14 days, to optimally detect healing fractures. The following implemented changes have led to valuable learning which provides opportunity for change to other teams:

- Booking x-ray at time of first skeletal survey

- Radiology service responsible for making appointment

- Clear discussion and expectation of social worker and carers to attend second appointment 